\title{
Mensagens da Prática Pedagógica sobre Educação Combinatória no contexto do EBRAPEM
}

\author{
Messages of the Pedagogical Practice about Combinatorial Education in the EBRAPEM \\ Context
}

\author{
Reinaldo Feio Lima
}

Universidade Federal do Sul e Sudeste do Pará, Faculdade de Matemática, PA, Brasil

E-mail: reinaldo.lima@unifesspa.edu.br

\begin{abstract}
Resumo
Este estudo objetiva identificar e compreender mensagens da prática pedagógica representadas em textos do EBRAPEM, com enfoque na Educação Combinatória, buscando responder: Qual(is) é(são) a(s) mensagem(ns) da prática pedagógica representadas em textos do EBRAPEM na perspectiva da Educação Combinatória? Trata-se de uma pesquisa qualitativa de caráter bibliográfico, a partir de pesquisas sobre "Educação Combinatória", disponíveis nos anais do EBRAPEM, compreendidas entre 2015 e 2019. Os textos publicados nesse período foram selecionados por meio dos seguintes descritores: combinatória, análise combinatória e educação combinatória. Foram identificadas 02 teses e 12 dissertações. Os dados foram analisados por meio da Análise de Conteúdo, a qual nos proporcionou constituir duas categorias, a priori: 1) aspectos físicos dos textos selecionados; 2) mensagem da prática pedagógica implícita nos textos selecionados. Os resultados mostraram, ao menos, duas mensagens: mensagem no campo da formação, que é caracterizada por pesquisas envolvendo professores como participantes de formação inicial e/ou continuada para uso da Educação Combinatória, com atividades referentes aos conhecimentos específicos, especializados e pedagógicos da combinatória planejada pelo pesquisador/formador. A outra mensagem compreendida neste artigo é a mensagem no campo da prática, que está relacionada às ações dos professores em sala ao implementarem a Educação Combinatória com atividades pedagógicas, tendo estudantes como protagonistas.
\end{abstract}

Palavras-chave: Mensagem. Prática Pedagógica. Educação Combinatória. EBRAPEM.

\begin{abstract}
This study aim to identify and understand messages of the pedagogical practices represented in EMBRAPEM texts, focusing on Combinatorial Education, seeking to answer: What is (are) the message(s) of the pedagogical practices represented in EBRAPEM in the perspective of Combinatorial Education? It is about a qualitative search of bibliographic character, from research on "Combinatorial Education" available in the EBRAPEM proceedings between 2015 and 2019. The texts published in this period were selected through the following descriptors: combinatorial, combinatorial analysis and combinatorial education. Two thesis and twelve dissertations were identified. The data were analyzed through the Content Analysis, which provided us to constitute two categories a priori: 1) physical aspects of the selected texts; 2) message of the pedagogical practice implicit in the selected texts. The results showed at least two messages: message in the field of teacher education, which is characterized by researches involving teachers as participants of the initial and/or continuing for the use of the Combinatorial Education, with activities referring to the specific, specialized and pedagogical knowledge of the Combinatorial planned by the researcher/training. Another message constant in this article is the message in the practice field, which is related to the teacher's actions in the classroom when implementing the Combinatorial Education with pedagogical activities having students as protagonists.
\end{abstract}

Keywords: Message. Pedagogical Practice. Combinatorial Education. EBRAPEM.

\section{Introdução}

Temos por objetivo, neste artigo, identificar e compreender a mensagem da prática pedagógica representada em textos dos anais das edições do Encontro Brasileiro de Estudantes de Pós-Graduação em Educação Matemática (EBRAPEM), com enfoque na Educação Combinatória (EC). Para alcançar o objetivo proposto, realizamos uma revisão bibliográfica, que se insere no contexto dos estudos metodológicos denominados 'Estado da Arte' ou 'Estado do Conhecimento', dos textos constantes nos anais das cinco últimas edições do EBRAPEM. Sobre o tema EC, foram levantadas e sistematizadas pesquisas publicadas no portal do EBRAPEM, entre 2015 e 2019, pois o intuito foi verificar como as produções científicas brasileiras recentes expressam a temática em questão. Compreendemos textos como os artigos publicados nos anais do EBRAPEM, porque são formas de representação visíveis, que comunicam alguma coisa, sejam elas expressas por meio da fala, escrita, visual etc (Bernstein, 2000).

Segundo Ferreira (2002), as pesquisas denominadas 'Estado da Arte' ou 'Estado do Conhecimento' são definidas como de caráter bibliográfico e possuem o propósito de mapear e analisar determinada produção acadêmica. Este artigo adota essa denominação, pois se circunscreve aos textos publicados no Encontro Brasileiro de Estudantes de Pós-Graduação em Educação Matemática (EBRAPEM), que tratam da prática pedagógica sobre Educação Combinatória. Dessa forma, prática pedagógica é conceituada por Bernstein (2000) de uma maneira ampla, como as relações sociais onde há alguém que está na condição de ensinar e alguém que está na condição 
de aprender. Por exemplo, entre formadores e professores ou professores e estudantes para ensinar e aprender determinado conteúdo.

Ao supormos que os textos publicados nos anais do EBRAPEM podem representar uma determinada prática pedagógica, podemos argumentar que mensagens dessa prática pedagógica podem se tornar coletivas por meio de um processo de comunicação/colaboração. A mensagem é o contexto da realização da prática pedagógica, ou seja, a relação entre alguém que está na condição de ensinar e alguém que está na condição de aprender, comunicando sobre Educação Combinatória, isto é, como pode falar, comunicar naquele contexto educacional (Bernstein, 2000). Compreendemos Educação Combinatória como um ramo da Educação Estatística que se ocupa da investigação de situações-problema relacionadas ao ensino e à aprendizagem de conceitos e procedimentos combinatórios.

Para alcançar tal "Estado do Conhecimento" ou "Estado da Arte", este artigo assume o paradigma construtivista, implicando uma ontologia relativista e uma epistemologia subjetivista, de modo que os resultados advindos das categorias, a priori, são construídos à medida que a apresentação e discussão dos dados se desenvolvem. Sob essa perspectiva, o pesquisador e o objeto da pesquisa assumem ligação interativa, isto é, numa relação estreita entre ontologia e epistemologia (Guba \& Lincoln, 1994).

OlevantamentofoirealizadonositedoEBRAPEM.Aescolha do site como locus se deve ao fato de reunir e disponibilizar produções científicas de abrangência nacional de mestrandos e doutorandos dos cursos de Pós-Graduação de grande importância para a promoção da pesquisa científica no Brasil.

A motivação para a realização deste artigo surgiu considerando-se a participação do autor na edição XXIII EBRAPEM. Em meio às discussões que ocorreram no grupo, um dos componentes indicou-nos a relevância de sintetizar os resultados das pesquisas sobre Educação Combinatória que foram obtidos pelas edições do EBRAPEM. Isso nos inspirou a realizar a pesquisa aqui apresentada.

Todas as questões serão desenvolvidas com o intuito de responder à questão: Qual é a(s) mensagem(ns) da prática pedagógica representada(s) em textos do EBRAPEM com enfoque na Educação Combinatória? Assim, a etapa foco deste artigo apresenta os resultados de um levantamento bibliográfico de pesquisas que se concentrou na Educação Combinatória, buscando indícios de práticas pedagógicas implementadas por professores que ensinam Matemática.

\section{Percurso Metodológico: da Constituição do Corpus de Pesquisa aos Procedimentos de Análises dos Dados}

O percurso metodológico deste artigo se adequa ao método qualitativo (MINAYO, 2010), de natureza bibliográfica, que, segundo Gil (2017), do tipo "Estado da Arte" ou "Estado do Conhecimento", pois tem o propósito de "[...] mapear e de discutir uma certa produção acadêmica em diferentes campos do conhecimento, tentando responder que aspectos e dimensões vêm sendo destacados e privilegiados em diferentes épocas e lugares" (Ferreira, 2002, p.258). O estudo foi realizado nos meses de janeiro e fevereiro de 2020, junto aos anais das cinco últimas edições do Encontro Brasileiro de Estudantes de Pós-Graduação em Educação Matemática.

Os textos publicados entre os anos 2015 e 2019 foram selecionadas por meio dos seguintes descritores: combinatória, análisecombinatóriaeeducaçãocombinatória,pois“osdescritores representam as palavras ou expressões que foram identificadas pelos autores das produções acadêmicas como representativo de sua pesquisa e, logo, possibilitam aos leitores identificar que o estudo contempla o tema em busca" (Amaral, Rosa, \& Locatelli, 2019, p.328). A partir da leitura dos títulos e dos resumos disponíveis no portal, buscaram-se palavras relacionadas com os descritores. Ao final desse processo, foram identificados 14 textos que atendiam aos descritores, formando nosso corpus da investigação. O Quadro 1 apresenta os textos selecionados com edição, ano, título, autor e instituição.

Quadro 1: Distribuição dos textos com enfoque na Educação Combinatória

\begin{tabular}{|c|c|c|c|c|c|}
\hline Evento & \begin{tabular}{|c|} 
Edição/ \\
Ano
\end{tabular} & Título & Autor(es) & Instituição & Código \\
\hline \multirow{5}{*}{ EBRAPEM } & \multirow{3}{*}{$\begin{array}{l}\mathrm{XIX} / \\
2015\end{array}$} & $\begin{array}{l}\text { As estratégias desenvolvidas por estudantes do } 2^{\circ} \text { ano } \\
\text { do Ensino Médio, antes e depois de terem estudado, em } \\
\text { ambiente escolar, o conteúdo de análise combinatória. }\end{array}$ & $\begin{array}{l}\text { Taianá Silva Pinheiro; } \\
\text { Sandra Maria Pinto } \\
\text { Magina. }\end{array}$ & UESC & A1 \\
\hline & & $\begin{array}{l}\text { A combinatória: abordagem em documentos oficiais, em } \\
\text { pesquisas e em livros didáticos. }\end{array}$ & $\begin{array}{l}\text { Monalisa Cardoso Silva; } \\
\text { Cristiane Azêvedo dos } \\
\text { Santos Pessoa } \\
\end{array}$ & UFPE & $\mathrm{A} 2$ \\
\hline & & $\begin{array}{l}\text { Aulas de combinatória no Ensino Médio: como estão } \\
\text { ocorrendo? }\end{array}$ & $\begin{array}{l}\text { Itatiane Borges Lima; } \\
\text { Cristiane Azevêdo dos } \\
\text { Santos Pessoa }\end{array}$ & UFPE & A3 \\
\hline & \multirow{2}{*}{$\begin{array}{l}\mathrm{XX} / \\
2016\end{array}$} & $\begin{array}{l}\text { Raciocínios combinatório e probabilístico na EJA: } \\
\text { investigando relações. }\end{array}$ & $\begin{array}{l}\text { Ewellen Tenório de Lima; } \\
\text { Rute Elizabete } \\
\text { de Souza Rosa Borba. } \\
\end{array}$ & UFPE & A4 \\
\hline & & $\begin{array}{l}\text { Conhecimento docente de combinatória e aluno: um caso } \\
\text { no interior de Pernambuco. }\end{array}$ & $\begin{array}{l}\text { Dorghisllany Souza } \\
\text { Holanda; Rute Elizabete } \\
\text { de Souza Rosa Borba }\end{array}$ & UFPE & A5 \\
\hline
\end{tabular}




\begin{tabular}{|c|c|c|c|c|c|}
\hline Evento & $\begin{array}{c}\text { Edição/ } \\
\text { Ano }\end{array}$ & Título & Autor(es) & Instituição & Código \\
\hline & \multirow{2}{*}{$\begin{array}{l}\text { XXI / } \\
2017\end{array}$} & $\begin{array}{l}\text { Análise de situações de ensino que envolvam raciocínio } \\
\text { combinatório no Ensino Fundamental. }\end{array}$ & $\begin{array}{l}\text { Daniela da Rosa Teza; } \\
\text { Maria Lucia } \\
\text { Panossian. }\end{array}$ & UFPR & A6 \\
\hline & & $\begin{array}{l}\text { Análise combinatória: um estudo de investigação com o } \\
\text { auxílio de jogos. }\end{array}$ & $\begin{array}{l}\text { Gabriel de Souza } \\
\text { Pinheiro; }\end{array}$ & UFRGS & A7 \\
\hline & \multirow{4}{*}{$\begin{array}{c}\mathrm{XXII} / \\
2018\end{array}$} & $\begin{array}{l}\text { A articulação entre combinatória e probabilidade nas } \\
\text { diferentes instâncias do currículo dos anos finais do } \\
\text { Ensino Fundamental: o que é dito, o que é feito e o que } \\
\text { se pode fazer? }\end{array}$ & $\begin{array}{l}\text { Ewellen Tenório de Lima; } \\
\text { Rute Elizabete de Souza } \\
\text { Rosa Borba. }\end{array}$ & UFPE & A8 \\
\hline & & $\begin{array}{l}\text { Um estudo exploratório e interventivo sobre } \\
\text { conhecimentos iniciais de combinatória na educação } \\
\text { infantil. }\end{array}$ & $\begin{array}{l}\text { Ariedja de Carvalho } \\
\text { Silva; Rute Elizabete de } \\
\text { Souza Rosa Borba. }\end{array}$ & UFPE & A9 \\
\hline & & $\begin{array}{l}\text { Resolução de problemas combinatórios nos anos iniciais: } \\
\text { uso do software PIXTON@. }\end{array}$ & $\begin{array}{l}\text { Dacymere Gadelha; Rute } \\
\text { Elizabete de Souza Rosa } \\
\text { Borba. }\end{array}$ & UFPE & A10 \\
\hline & & $\begin{array}{l}\text { Modelagem na educação matemática para o } \\
\text { desenvolvimento de conceitos de análise combinatória. }\end{array}$ & $\begin{array}{l}\text { Ticiano Azevedo Bastos; } \\
\text { Milton Rosa }\end{array}$ & UFOP & A11 \\
\hline & \multirow{3}{*}{$\begin{array}{c}\text { XXIII / } \\
2019\end{array}$} & $\begin{array}{l}\text { Os currículos moldado, realizado e avaliado: } \\
\text { perspectivas de professores sobre articulações entre } \\
\text { combinatória e probabilidade. }\end{array}$ & $\begin{array}{l}\text { Ewellen Tenório de Lima; } \\
\text { Rute Elizabete de Souza } \\
\text { Rosa Borba }\end{array}$ & UFPE & A12 \\
\hline & & $\begin{array}{l}\text { Tarefas de análise da produção escrita para o ensino e } \\
\text { aprendizagem de análise combinatória. }\end{array}$ & $\begin{array}{l}\text { Erika Regina Santana } \\
\text { da Silva Pereira; Jader } \\
\text { Otávio Dalto }\end{array}$ & UTFPR & A13 \\
\hline & & $\begin{array}{l}\text { Estocástica no } 2^{\circ} \text { ano dos anos iniciais do Ensino } \\
\text { Fundamental: uma possibilidade. }\end{array}$ & $\begin{array}{l}\text { Sezilia Elizabete } \\
\text { Rodrigues Garcia } \\
\text { Olmo de Toledo; Celi } \\
\text { Espasandin Lopes }\end{array}$ & UNICSUL & A14 \\
\hline
\end{tabular}

Fonte: Dados da pesquisa.

Dessa forma, já com os textos selecionadas, iniciouse o processo de análise por meio da Análise de Conteúdo que, segundo Bardin (2011, p.24), “é um conjunto de instrumentos metodológicos cada vez mais sutis em constante aperfeiçoamento, que se aplicam a discursos" ou ainda "uma técnica de investigação que tem por finalidade a descrição objetiva, sistemática e recorrente do conteúdo manifesto na comunicação" (Bardin, 2011, p.24), considerando as seguintes etapas (Bardin, 2011): 1) Pré-análise, triagem e organização do material a ser pesquisado por meio de "leitura flutuante" dos textos. Neste momento, realizamos a leitura de todos os textos publicados nos anais do EBRAPEM para constituir o corpus, obedecendo aos critérios precisos de escolha; 2) Exploração do material das operações de codificação, isto é, operações de recorte e enumeração das informações textuais, as quais são identificadas por meio dos descritores que, ao serem agregadas, constituem as categorias a priori; 3 ) Tratamento dos dados, realizou-se a análise das categorias a priori, interpretadas à luz do objetivo e conhecimentos já desenvolvidos em torno da temática. Organizamos todas as informações em um fichamento que se constituiu nosso instrumento de análise. A partir da organização das informações, primeiramente, definimos duas categorias: 1) aspectos físicos dos textos selecionados; 2) mensagem da prática pedagógica implícita nos textos selecionados. Tais categorias, de início, às quais nos dedicamos a desenvolver na próxima seção, se constituem nosso instrumento de apresentação dos dados (Araújo, 2013).

\section{Apresentação e Discussão dos Dados}

Nesta seção, organizada em duas partes, são apresentadas as categorias a priori que foram consideradas para sistematizar os 14 textos que fizeram parte do corpus. Em cada uma delas, o leitor encontrará algumas aproximações e discussões sobre a temática Educação Combinatória recuperadas no site do EBRAPEM, elaboradas pelo autor deste artigo.

\subsection{Aspectos Físicos dos Textos Selecionados}

Podemos observar, com base no Quadro 1, uma oscilação na quantidade de artigos publicados a partir de 2015. A média entre os anos de 2015 e 2020 foi de menos de 3 publicações por ano, ou seja, a quantidade de publicações de artigos de 2011 a 2018, que é de 14, representa poucas pesquisas com foco na Educação Combinatória. Conclui-se, portanto, que as pesquisas acerca da EC têm oscilado com o passar dos anos. Esse quantitativo está assim distribuído: três trabalhos da edição do EBRAPEM de 2015, dois de 2016, dois de 2017, quatro de 2018 e três de 2019. Isso nos dá indícios de que o(a) professor(a) e os grupos de pesquisas estão cada vez mais produzindo pesquisas com enfoque nos conceitos de Probabilidade e Estatística. Estas, por sua vez, têm, cada 
vez mais, ganhado espaço entre os conteúdos que deverão ser ensinados na educação básica, tanto quanto a Geometria, a Álgebra e a Aritmética (Meneghetti, Batistela, \& Bicudo, 2011).

Sobre a origem dos autores dos trabalhos, o que se observa é que não há um núcleo de pesquisa que se destaca, pois, dos quatorze textos, oito são da Universidade Federal de Pernambuco (UFPE) e todos os demais são de origens diferentes, a saber: Universidade Estadual de Santa Cruz (UESC); Universidade Federal do Paraná (UFPR); Universidade Federal do Rio Grande do Sul (UFRGS); Universidade Federal de Ouro Preto (UFOP); Universidade Tecnológica Federal do Paraná (UTFPR) e Universidade Cruzeiro do Sul (UNICSUL).

Esses trabalhos foram produzidos, basicamente, nos seguintes programas de Pós-Graduação, a saber: Programa de Pós-Graduação em Educação Matemática e Tecnológica (EDUMATEC) da Universidade Federal de Pernambuco (UFPE), com oito textos; e os demais com um texto cada, Programa de Pós-Graduação em Educação Matemática (PPGEM), da Universidade Estadual de Santa Cruz (UESC); Programa de Pós-Graduação em Educação em Ciências e Matemática (PPGECM), da Universidade Federal do Paraná (UFPR); Programa de Pós-Graduação em Ensino de Matemática (PPG-EMAT), da Universidade Federal do Rio Grande do Sul (UFRGS); Programa de Pós-Graduação em Educação Matemática (PPGEDMAT), da Universidade Federal de Ouro Preto (UFOP); Programa de Pós-Graduação em Formação Científica, Educacional e Tecnológica (PPGFCET), da Universidade Tecnológica Federal do Paraná (UTFPR); e Programa de Pós-Graduação em Ensino de Ciências e Matemática (PPECM), da Universidade Cruzeiro do Sul (UNICSUL).

Nas modalidades dos cursos de pós-graduação stricto sensu, mestrados e doutorados, foram encontradas duas dissertações de Mestrado Profissional (A7 e A11), dez de Mestrado Acadêmico (A1, A2, A3, A4, A5, A6, A9, A10, A13 e A14) e duas teses de Doutorado (A8 e A12) versando sobre Educação Combinatória.

Tratando do referencial teórico para embasamento da Educação Combinatória, elencamos os principais referenciais teóricos dos 14 textos selecionados. O referencial teórico principal com maior incidência foi a Teoria dos Campos Conceituais em seis deles, dentre os 14 textos. Dois textos se fundamentam nas ideias de Sacristán. Dois textos mencionam como referencial teórico os trabalhos de Shulman e Skovsmose, respectivamente. Nos demais, são tomados como referenciais discussões de outros trabalhos.

Todas os textos selecionados estão ancorados no método qualitativo. Os instrumentos de produção de dados mais utilizados foram questionários e entrevistas semiestruturadas, mas também se utilizaram gravações em áudio-vídeo, observação, além de análise documental e bibliográfica.

Com relação ao nível de ensino dos textos, pode- se identificar um maior interesse dos pesquisadores em investigar a Educação Combinatória no Ensino Fundamental e Médio, com cinco textos publicados em cada nível, seguido da Educação Infantil, Ensino Superior, Educação de Jovens e Adultos, com uma publicação cada. Entre as pesquisas, apenas uma se desenvolveu em dois níveis de ensino: Ensino Fundamental e Médio.

Quanto aos orientadores desses textos selecionados, os dados indicaram que todos são pesquisadores doutores, trabalham em instituições públicas e são orientadores de dissertações e teses. São ele: Rute Elizabete de Souza Rosa Borba, com 6 textos orientados, Cristiane Azevedo dos Santos Pessoa, com dois textos, e os demais cada um com um texto orientado, por exemplo, Celi Espasandin Lopes, Jader Otávio Dalto, Maria Lucia Panossion, Marilaine de Fraga Sant'Ana, Milton Rosa e Sandra Maria Pinto Magina.

\subsection{Mensagens da prática pedagógica implícitas nos textos selecionados}

Prática pedagógica envolvendo professores como sujeitos participantes foram identificadas em dois textos (A3 e A5). Silva e Pessoa (2015) tinham como objetivo analisar o conhecimento docente sobre combinatória atuante em turmas de $2^{\circ}$ ano de Ensino Médio. Os sujeitos participantes da pesquisa foram três professores de escolas da rede pública de ensino da região metropolitana de Recife. Por meio dos instrumentos de produção de dados (entrevistas e observações), analisaram as situações (tipos de problemas); os invariantes explicitados ao ensinar; as representações utilizadas e estimuladas durante as aulas e investigar o Conhecimento Pedagógicos do Conteúdo que esses professores apresentam sobre combinatória. Esses dados seriam analisados à luz da Teoria de Vergnaud (1986) e dos estudos Ball et al (2008).

Holanda e Borba (2015) propõem-se a analisar como são mobilizados conhecimentos docentes a partir do conhecimento de combinatória e alunos, em aulas de um curso de formação inicial de professores de Matemática. Para alcançar o objetivo traçado, foram observadas 15 aulas do componente curricular "Ensino de Combinatória: perspectivas teóricas e práticas", do curso de Licenciatura em Matemática do Centro Acadêmico do Agreste, em Caruaru, Pernambuco, sendo realizadas anotações e gravações de áudio e vídeo das aulas. Os resultados iniciais indicaram as relações com o conhecimento de combinatória e aluno. A professora formadora, em diversos momentos, destacou a importância desse tipo de conhecimento, preocupando-se com as necessidades dos estudantes, tanto no que se refere ao conhecimento do conteúdo específico, quanto ao conhecimento do ensino de combinatória.

Em suma, os autores ainda ressaltam que, segundo a perspectiva Vygotskyana (2001), a interação social possui um papel fundamental no desenvolvimento cognitivo e no desenvolvimento cultural de um sujeito. Portanto, ela é origem e motor da aprendizagem e do desenvolvimento intelectual. Além disso, os estudos apontam para uma lacuna nos anais 
do EBRAPEM, em que fica evidenciada pouca atenção na formação de professores em formação inicial e continuada com enfoque na Educação Combinatória. Essa lacuna, também, foi realçada nos estudos de Fernandes, Correia e Roa (2010), que destacam haver pouca exploração de investigações didáticas sobre combinatória e probabilidade.

A nosso ver, o contexto colaborativo/participativo e o percurso do grupo de estudos podem suprir as lacunas na formação docente, possibilitando a ampliação do conhecimento combinatório. Do mesmo modo, o fomento de comunidades de prática também evoca o estabelecimento de ações de formação que promovem experiências que priorizam a construção, compreensão, apreensão e reprodução de procedimentos, conceitos e ideias que possam estar direcionados para Educação Combinatória (Estevam \& Cyrino, 2016; Conti, 2016; 2018).

Guirado e Cardoso (2007) consideram que materiais manipulativos, juntamente com a construção de esquemas, tabelas, diagramas ou desenhos, possibilitam o desenvolvimento da Educação Combinatória. Nesse aspecto, o ensino significativo da combinatória é sugerido por Rocha (2011), argumentando que os professores que ensinam Matemática devem compreender os níveis de conhecimento de estruturas combinatórias, os conhecimentos dos seus estudantes e suas estratégias de resolução de situaçõesproblema.

Dessa forma, podemos inferir que a mensagem da prática pedagógica sobre Educação Combinatória envolvendo professores presente nos textos do EBRAPEM pode estar vinculada à mensagem no campo da formação. Esta, por sua vez, reforça a necessidade de ampliar a discussão sobre o campo de formação (inicial, continuada ou exercício) de professores que lecionam Matemática nos diferentes níveis de ensino.

Prática pedagógica envolvendo estudantes como sujeitos participantes foram identificadas em 12 textos (A1, A2, A4, A6, A7, A8, A9, A10, A11, A12, A13, A14), que descreveremos a seguir.

Os estudos representados nos textos A1, A6, A9 e A13 mostram práticas pedagógicas desenvolvidas com estudantes em diferentes níveis de ensino. As pesquisas de Pinheiro e Magina (2015) e Pereira e Dalto (2019) convergiram para os mesmos sujeitos participantes, ambos focaram em estudantes do Ensino Médio. Os estudos de Pinheiro e Magina (2015) analisaram as estratégias desenvolvidas por estudantes do $2^{\circ}$ ano do Ensino Médio de uma escola pública do extremosul da Bahia, antes e depois de terem estudado, em ambiente escolar, o conteúdo de Análise Combinatória. Os resultados iniciais indicaram que, através da análise das resoluções dos problemas dos alunos, é possível perceber como se dá o raciocínio combinatório dos mesmos e, assim, contribuir para a ressignificação do ensino e a aprendizagem de Análise Combinatória. Já a pesquisa de Pereira e Dalto (2019) teve por objetivo investigar a utilização da análise da produção escrita como estratégia de ensino para a aprendizagem do conteúdo de Análise Combinatória para alunos do Ensino Médio. Os resultados desse trabalho podem contribuir para a aprendizagem do conteúdo de Análise Combinatória e para Educação Matemática.

Enquanto isso, Teza e Panossian (2017) analisaram a influência do processo de organização do ensino sobre o desenvolvimento do raciocínio combinatório dos estudantes nos anos finais do Ensino Fundamental. Já Silva e Borba (2018) tiveram como objetivo desenvolver uma investigação junto a crianças da Educação Infantil, no sentido de sondar quais aspectos do raciocínio combinatório já se encontram em processo inicial de construção e quais atividades podem ser desenvolvidas junto aos alunos no início da escolarização, de maneira a ampliar o desenvolvimento de seus raciocínios combinatórios.

Analisando as práticas pedagógicas desses quatro textos acerca da temática em questão, percebe-se que o foco principal está na aprendizagem do estudante. E que esta aprendizagem estava diretamente relacionada ao objetivo de levar o aluno a lidar com situações-problema que envolvam combinações, arranjos, permutações e, especialmente, o princípio multiplicativo da contagem (Brasil, 1998). Em relação à Análise Combinatória, na Base Nacional Comum Curricular - BNCC, é proposta a progressão ano a ano, a partir da "compreensão e utilização de novas ferramentas e também na complexidade das situações-problema propostas, cuja resolução exige a execução de mais etapas ou noções de unidades temáticas" (Brasil, 2018, p.277). Podemos inferir que, em ambos os documentos, é direcionada a ideia de desenvolver no estudante a capacidade de articular conteúdos vistos na escola ao seu dia a dia. Os alunos se relacionam com os objetos combinatórios e as práticas cotidianas. Então, é necessário que o docente aborde em suas aulas exemplos vivenciados no dia a dia pelo estudante para que o saber tenha sentido/significado. Nessa perspectiva, ensinar é

[...] mobilizar a atividade dos alunos para que construam saberes e transmitir-lhes um patrimônio de saberes sistematizados legados pelas gerações anteriores de seres humanos. Conforme os aportes de Bachelard, o mais importante é entender que a aprendizagem nasce do questionamento e leva a sistemas constituídos. É essa viagem intelectual que importa. Ela implica que o docente não seja apenas professor de conteúdos, isto é, de respostas, mas também, e em primeiro lugar, professor de questionamento. Quanto aos alunos, às vezes, andarão sozinhos, com discreto acompanhamento da professora e, outras vezes, caminharão com a professora de mãos dadas. O mais importante é que saibam de onde vêm, por que andam e, ainda, que cheguem a algum lugar para o qual valha a pena ter feito a viagem (Charlot, 2014, p.53).

Corroborando essa assertiva, a aprendizagem combinatória deve estar relacionada com a vivência do estudante, para que, assim, o conteúdo passe a ter sentido e significado. Isso significa dizer que é preciso que a prática pedagógica 
do professor que ensina Matemática esteja alicerçada no sentido de não dar respostas prontas aos questionamentos que emergem do processo de aprendizagem, mas que o leve a pensar, criticar, questionar o enunciado do problema combinatório e, extrapolando os muros da escola, no sentido de buscar respostas para que todo o saber tenha um sentido. Nessa perspectiva, o fato de aprender determinado conteúdo combinatório está diretamente ligado ao sentido e significado que damos pela forma como o docente o apresenta, para que, assim, os estudantes possam se apropriar dos saberes, configurando mensagem de aprendizagem.

Percebemos uma convergência entre os trabalhos analisados, uma vez que A7, A10 e A11 focaram na prática pedagógica com estudantes fazendo uso de jogos. A7 (Pinheiro, 2017) experimentou uma proposta de sequência didática para o ensino de Análise Combinatória no Ensino Médio, utilizando os jogos em sala de aula como suporte para a construção dos conceitos. A10 (Gadelha \& Borba, 2018) analisou o quanto as resoluções ilustradas podem contribuir para a compreensão de situações combinatórias, com 36 estudantes do $5^{\circ}$ ano do Ensino Fundamental usando o recurso didático software Pixton $($.

Enquanto isso, A11 (Toledo \& Lopes, 2019) buscou descrever e compreender os indícios de constituição das distintas formas de raciocínio combinatório, probabilístico e estatístico apresentadas pelas crianças na resolução de um problema matemático fazendo uso de jogos. Os resultados revelaram que as crianças passaram a ter um olhar mais atento para as apostas em jogos com dados, a reconhecer gráfico de colunas e a usar termos pertinentes à Estatística, como "chance, probabilidade, impossível, provável".

Os resultados desses três estudos reforçam o argumento potencial do uso de jogos e recursos tecnológicos na prática pedagógica do professor que ensinam Matemática, como forma de auxiliar na aprendizagem de conteúdos combinatórios. Isso estimula avanços no pensamento lógico-dedutivo e também é uma maneira de representar, contribuindo para a abrangência de outras representações simbólicas válidas na resolução de problemas de combinatória, como apontam os resultados do estudo de Gadelha e Montenegro (2018), Gadelha; Vicente; Montenegro (2017), e Rodrigues, Souza e Lucena (2016), caracterizando, assim, mensagem com uso de jogos e recursos tecnológicos.

Dos textos selecionados, apenas o de Lima e Borba (2016) apresenta resultados de um estudo com discentes da Educação de Jovens e Adultos (EJA). O único que traz uma análise da aprendizagem dos estudantes num ambiente de modelagem matemática é o de Bastos e Rosa (2018).

Outra característica ímpar nos trabalhos apresentados é a pesquisa documental sobre os documentos curriculares do Brasil, realizada por Silva e Pessoa (2015), Lima e Borba (2018; 2019). Silva e Pessoa (2015) analisaram a abordagem da combinatória em orientações curriculares de documentos oficiais, em resultados de pesquisas e em livros didáticos de
Matemática destinados à Educação Básica. Essa análise foi realizada à luz da Teoria dos Campos Conceituais (Vergnaud, 1986), que embasa o olhar sobre o livro didático, no sentido de observar se as dimensões do conceito estão presentes nos livros analisados. Já Lima e Borba $(2018 ; 2019)$ aprofundaram o entendimento das relações entre Combinatória e Probabilidade a partir da investigação de evidências/ indicações da existência e importância delas nas diferentes instâncias do currículo referente aos anos finais do Ensino Fundamental. E, posteriormente, levantar informações acerca do currículo moldado, do currículo em ação e do currículo avaliado. Os resultados preliminares levantados pelos estudos citados reforçam a realização da pesquisa documental, a fim de identificar as limitações dos livros didáticos e documentos curriculares para subsidiar a futura prática pedagógica e as concepções sobre o ensino de combinatória para a educação básica, caracterizando, assim, mensagem diagnóstica.

$\mathrm{O}$ conjunto das mensagens identificadas na prática pedagógica envolve estudantes como sujeitos participantes: mensagem de aprendizagem, mensagem com uso de jogos e recursos tecnológicos e mensagem diagnóstica. É possível, a partir da análise realizada neste estudo, sugerir outra mensagem. Os dados indicam que a mensagem sobre Educação Combinatória comunicada na prática pedagógica envolvendo estudantes no contexto do EBRAPEM é mensagem no campo da prática e se atém ao espaço da sala de aula.

\section{Conclusões elimplicações}

Nosso propósito foi identificar e compreender a mensagem da prática pedagógica representada em textos dos anais das edições do Encontro Brasileiro de Estudantes de Pós-Graduação em Educação Matemática (EBRAPEM), com enfoque na Educação Combinatória (EC). Nesse sentido, compreendemos prática pedagógica como as relações entre aquele que está na condição de ensinar e aquele que está na condição de aprender nos diferentes espaços escolares (Bernstein, 2000).

Nas conclusões das pesquisas quanto ao desenvolvimento da Educação Combinatória representada nos 14 textos que compõem o corpus da investigação, identificamos duas mensagens da prática pedagógica: mensagem no campo da formação e mensagem no campo da prática. Tais mensagens nos permitiram identificar prática pedagógica envolvendo professores e estudantes como sujeitos participantes no processo de ensino e de aprendizagem da Educação Combinatória.

Nesse sentido, destacamos a mensagem no campo da formação, que é caracterizada por pesquisas envolvendo professores como participantes de formação inicial e/ou continuada para uso da Educação Combinatória, com atividades referentes aos conhecimentos específicos, especializados e pedagógicos da combinatória planejada pelo pesquisador/ formador. Em geral, nessa mensagem no campo da formação 
repousa sua preocupação, essencialmente, o "como ensinar". O como ensinar foi compreendido como uma expectativa da realização contextual de uma prática pedagógica em que professor possa constantemente (re)modelar uma pedagogia eficaz. O como ensinar também foi contemplado nos artigos que fizeram parte desta categoria, sendo discutido a partir do contexto de uma situação-problema para investigar o conhecimento pedagógico do conteúdo que esses professores apresentam sobre combinatória (Silva \& Pessoa, 2015) e o componente curricular "Ensino de Combinatória: perspectivas teóricas e práticas" do curso de Licenciatura em Matemática (Holanda \& Borba, 2015). A partir daí, foram apontadas contribuições destas propostas para a formação do professor de Matemática.

Outra mensagem compreendida neste artigo é a mensagem no campo da prática, que está relacionada às ações pedagógicas dos professores em sala ao implementarem a Educação Combinatória com atividades pedagógicas, tendo estudantes como protagonistas. De modo geral, a ação pedagógica do professor sobre tal perspectiva busca provocar nos estudantes a reflexão sobre o que ele está fazendo ao longo do processo de resolução de situações-problema-combinatórias (Serrazina, 2017). Recentemente, a Base Nacional Comum Curricular (BNCC, 2018) também passou a prever, apresentando a relevância do ensino de conteúdo combinatório voltado para a produção e análise de ideias que devem estar alinhadas ao contexto da resolução de situações-problema.

As conclusões dessas pesquisas revelaram que o foco está na aprendizagem, pois, mediado pelo uso de estratégias pedagógicas, constituiu-se em oportunidades de melhorias na Educação Combinatória por meio de jogos e recursos tecnológicos, conforme apontam os estudos de Pessoa e Borba (2012). Eles observaram crianças da Educação Infantil resolvendo problemas combinatórios utilizando material manipulativo. Assim como apontam os estudos de Azevedo e Borba (2013), ao apresentarem resultados de estudantes resolvendo problemas combinatórios com uso de árvores de possibilidades - construídas em lápis e papel ou com auxílio de um software -, e Pessoa e Santos (2015) que trabalharam com o uso do material manipulável no processo de ensino da combinatória. Esses resultados comprovam que o uso de jogos e recursos tecnológicos potencializa as atividades envolvendo a Educação Combinatória.

Dessa forma, acreditamos que o trabalho desenvolvido com a Educação Combinatória desde a Educação Infantil, se não for bem explorado, pode ficar defasado, uma vez que, os documentos oficiais apontam para o trabalho com a Combinatória na escola desde o início do Ensino Fundamental. Além disso, os livros didáticos dos anos iniciais e dos anos finais do Ensino Fundamental direcionam para atividades/ tarefas matemáticas de situações-problemas combinatórios (Barreto; Amaral \& Borba, 2007).

Nesse sentido, observamos que são poucas as pesquisas de doutorado tratando do tema, dentre os 14 textos, apenas 2 são teses de doutorado elaboradas, ainda nesta década, com foco no tema da Educação Combinatória. Portanto, apresenta uma lacuna nesse campo de estudo e, ao mesmo tempo, se mostrou como um número preocupante uma vez que, em geral, trabalhos neste nível balizam outras pesquisas da comunidade científica, por exemplo, o foco recai no ensino de Probabilidade e Estatística.

As compreensões geradas por este artigo podem subsidiar as futuras práticas pedagógicas de professores que ensinam Matemática com enfoque no desenvolvimento da Educação Combinatória nas salas de aula. No que diz respeito às questões relacionadas à formação de professores - inicial e/ou continuada -, as compreensões abrem horizontes para futuras investigações sobre as práticas de docentes de Matemática em comunidade de práticas ou grupo colaborativo com o intuito de minimizar a lacuna na formação combinatória. Além disso, este estudo pode contribuir para o campo da Educação Matemática e Educação Estatística em trabalhos que discutam sobre prática pedagógica envolvendo professores e estudantes como sujeitos participantes.

Por fim, espera-se que este Estado do Conhecimento auxilie na proposição de outras pesquisas sobre Educação Combinatória na formação inicial e continuada de professores que ensinam Matemática na Educação Básica e no Ensino Superior, trazendo novos elementos, conhecimentos, saberes e discussões. A produção acadêmica brasileira é um campo inesgotável para pesquisas, fato que favorece o preenchimento das possíveis lacunas evidenciadas neste estudo e a concretização de outras, uma vez que acreditamos que este tipo de pesquisa é uma relevante fonte de consulta científica tanto para pesquisadores em início de carreira como pesquisadores com larga experiência no campo da Educação Estatística.

\section{Referências}

Amaral, L. Z., \& Rosa, C. T. W.; Locatelli, A. (2019). Educação em ciências/química e alfabetização científica na perspectiva da formação cidadã: características e tendências das pesquisas nacionais. Revista REAMEC, 7(3), 321-350.

Araujo, E. S. (2013). Contribuições da teoria histórico-cultural à pesquisa em Educação Matemática: a Atividade Orientadora de Pesquisa. Horizontes, 31(1), 81-90.

Azevedo, J., \& Borba, R. (2013). Combinatória: a construção de árvores de possibilidades por alunos dos anos iniciais com e sem uso de software. Alexandria Rev. Educ. Ciênc. Tecnol., 6(2), 113-140.

Ball, D. L., Thames, M.H., \& Phelps, G. (2008). Content Knowledge for Teaching: what makes it special? In: Journal of teacher educacion., 59(5), 389-407.

Barreto, F., Amaral, F., \& Borba, R. (2007). Como o raciocínio combinatório tem sido apresentado em livros didáticos de séries iniciais. Caderno de Trabalhos de Conclusão de Curso de Pedagogia, 2, 1-21.

Bardin, L. (2011). Análise de Conteúdo. São Paulo. Edições: 70.

Bastos, T. A., \& Rosa, M. (2018). Modelagem na educação matemática para o desenvolvimento de conceitos de análise 
combinatória. In: Anais do 19 Encontro Nacional de Educação Matemática (ENEM), Belo Horizonte, Minas Gerais, 2018.

Bernstein, B. (2000). Pedagogy, symbolic control and identity: theory, research, critique. New York: Rowman\& Littlefield.

Brasil. (2018). Ministério da Educação. Base Nacional Comum Curricular (BNCC). Brasília: MEC.

Brasil. (1998). Parecer CEB 04/98. Diretrizes Curriculares Nacionais para o Ensino Fundamental. Brasília: MEC.

Charlot, B. (2014). Da relação com saber às práticas educativas. São Paulo: Cortez.

Conti, K. C. (2016). Educação Estatística num contexto colaborativo: ensinar e aprender probabilidade. Educ. Matem. Pesq., São Paulo, 18(3), 1117-1140.

Conti, K. C. (2018). O estudo da estatística num contexto colaborativo: o gráfico de setores. REnCiMa, 9(2), 265-282.

Estevam, E. J. G., \& Cyrino, M. C. C. T. (2016). Comunidades de Prática como contexto para o desenvolvimento profissional docente em Educação Estatística. Educ. Matem. Pesq., São Paulo, 18(3)1291-1317.

Fernandes, J., Correia, P., \& Roa, R. (2010). Aquisição das operações combinatórias por alunos pré-universitários através de uma intervenção de ensino. Revista Latinoamericana de Investigación en Matemática Educativa, 13(2) 215242.

Ferreira, N. S. de A. (2002). As pesquisas denominadas "Estado da Arte". Educação \& Sociedade, Campinas, 23(79) 257-272.

Gadelha, C., \& Borba, R. E. S. R. (2018). Resolução de problemas combinatórios nos anos iniciais: uso do software PIXTON(C. In: Anais do 19 Encontro Nacional de Estudantes de Educação Matemática (ENEM), Belo Horizonte, Minas Gerais.

Gadelha, D., \& Montenegro, J. (2018). Interfaces das representações simbólicas por crianças antes e depois de um processo de intervenção em Combinatória. In: Anais Do 07 Vii Encontro De Pesquisa Educacional Em Pernambuco, Recife, Pernambuco.

Gadelha, D., Vicente. D., \& Montenegro J. (2017). Software Pixton(C): resolução de problemas combinatórios por crianças do $5^{\circ}$ ano do Ensino Fundamental. In: Cadernos de trabalho de conclusão do curso de graduação em Pedagogia da UFPE. Recife, 2017.

Gil, A. C. (2017). Como Elaborar Projetos de Pesquisa. 6 ed. São Paulo, Atlas.

Guba, E., \& Lincoln, Y. 1994. Competing paradigms in qualitative research. In: N.K. DENZIN e Y. LINCOLN (eds.), Handbook of qualitative research. Thousand Oaks, Sage Publications, (pp. 105-117).

Guirado, J. C., \& Cardoso, E. (2007). Análise combinatória: da manipulação à formalização de conceitos. In: Anais do 09 Encontro Paranaense de Educação Matemática. Paraná, Paraná.

Holanda, D. S., \& Borba, R. E. S. R. (2015). Conhecimento docente de combinatória e aluno: um caso no interior de Pernambuco. In: Anais XIX Encontro Nacional de Educação Matemática, Curitiba, Paraná

Lima, E. T., \& Borba, R. E. S. R. (2018). A articulação entre combinatória e probabilidade nas diferentes instâncias do currículo dos anos finais do ensino fundamental: o que é dito, o que é feito e o que se pode fazer? In: Anais do 19 Encontro Nacional de Educação Matemática (ENEM), Belo Horizonte, Minas Gerais.
Lima, E. T., \& Borba, R. E. S. R. (2019). Os currículos moldado, realizado e avaliado: perspectivas de professores sobre articulações entre combinatória e probabilidade. In: Anais do 19 Encontro Nacional de Educação Matemática, São Paulo, 2019 .

Lima, E. T., \& Borba, R. E. S. R. (2016). Raciocínios combinatório e probabilístico na EJA: investigando relações. In: Anais do 19 Encontro Nacional de Educação Matemática (ENEM), Curitiba, Paraná.

Lima, I. B., \& Pessoa, C. A. S. (2015). Aulas de combinatória no ensino médio: como estão ocorrendo?. In: Anais do 19 Encontro Nacional de Educação Matemática (ENEM), Juiz de Fora, Rio de Janeiro

Meneghetti, R. C. G., Batistela, R. F., \& Bicudo, M. A. V. (2011). A pesquisa sobre o ensino de probabilidade e estatística no brasil: um exercício de metacompreensão. Bolema, v. 24(40) 811-833.

Minayo. M. C. (2010). O desafio do conhecimento: pesquisa qualitativa em saúde. ed. 12. São Paulo: HUCITEC ABRASCO.

Pereira, E. R. S. S., \& Dalto, J. O. (2019). Tarefas de análise da produção escrita para o ensino e aprendizagem de análise combinatória. In: Anais do 19 Encontro Nacional de Educação Matemática, São Paulo.

Pessoa, C., \& Borba, R. (2012). Do young children notice what combinatorial situations require? In: Proceedings of the 36th Conference of the International Group for the Psychology of Mathematics Education (PME), Taipei (Taiwan).

Pessoa, C., \& Santos, L. T. (2015). Resolução de problemas combinatórios a partir de material manipulativo e de lápis e papel: intervenções no $5^{\circ}$ ano do ensino fundamental. Revista Educação Online, 18(1) 2015.

Pinheiro, G. S. (2017). Análise combinatória: um estudo de investigação com o auxílio de jogos. In: Anais do 19 XIX Encontro Nacional de Estudantes de Educação Matemática (ENEM), Pelotas, Rio Grande do Sul.

Pinheiro, T. S., \& Magina, S. M. P. (2015). As estratégias desenvolvidas por estudantes do $2^{\circ}$ ano do ensino médio, antes e depois de ter estudado, em ambiente escolar, o conteúdo de análise combinatória. In: Anais do 19 Encontro Nacional de Educação Matemática (ENEM), Juiz de Fora, Minas Gerais.

Rodrigues, H., Souza, R., \& Lucena, R. (2016). Funções matemáticas em quadrinhos: contextualização com o Pixton. In: Anais do 14 CONGRESSO INTERNACIONAL DE TECNOLOGIA NA EDUCAÇÃO (CITE), Pernambuco, Recife.

Rocha, C. (2011). Formação Docente e o Ensino de problemas combinatórios: diferentes conhecimentos, diversos olhares. (192 f.). Dissertação de Mestrado, Programa de Pós-Graduação em Educação Matemática e Tecnológica, Universidade Federal de Pernambuco, Recife.

Serrazina, L. (2017). Resolução de Problemas e Formação de Professores: um olhar sobre a situação de Portugal. In: ONUCHIC, L; JUNIOR, L; PIRONEL, M. (Orgs.). Perspectivas para a Resolução de Problemas. Ed. Livraria da Física, (pp.55-83).

Silva, A. C., \& Borba, R. E. S. R. (2018). Um estudo exploratório e interventivo sobre conhecimentos iniciais de combinatória na educação infantil. In: Anais do 19 Encontro Nacional de Estudantes de Educação Matemática (ENEM), Belo Horizonte, Minas Gerais. 
Silva, M. C., \& Pessoa, C. A. S. (2015). A combinatória: abordagem em documentos oficiais, em pesquisas e em livros didáticos. In: Anais do 19 Encontro Nacional de Educação Matemática (ENEM), Juiz de Fora, São Paulo.

Teza, D. R., Panossian, M. L. (2017). Análise de situações de ensino que envolvam raciocínio combinatório no ensino fundamental. In: Anais do 19 Encontro Nacional de Educação Matemática (ENEM), Pelotas, Rio Grande do Sul.

Toledo, S. E. R. G. O., \& Lopes, C. E. (2019). Estocástica no $2^{\circ}$ ano dos anos iniciais do ensino fundamental: uma possibilidade. In: Anais do 19 Encontro Nacional de Educação Matemática (ENEM), São Paulo.

Vergnaud, G. (1986). Psicologia do desenvolvimento cognitivo e didática das matemáticas. Um exemplo: as estruturas aditivas. Análise Psicológica, 1.

Vygotsky, L. S. (2001). Construção do pensamento e da linguagem. São Paulo: Martins Fontes. 